$\xi=1$ -

\title{
Early Detection of Diabetic Retinopathy by Using Deep Learning Neural Network
}

\author{
Mohamad Hazim Johari ${ }^{1}$, Hasliza Abu Hassan ${ }^{2}$, Ahmad Ihsan Mohd Yassin ${ }^{1}$, Nooritawati Md Tahir ${ }^{1}$, Azlee \\ Zabidi $^{1}$, Zairi Ismael Rizman ${ }^{3}$, Rahimi Baharom ${ }^{1}$, Norfishah Abdul Wahab ${ }^{1}$ \\ ${ }^{1}$ Faculty of Electrical Engineering, Universiti Teknologi MARA (UiTM), Shah Alam, Selangor, Malaysia \\ ${ }^{2}$ Faculty of Engineering and Life Sciences, Bestari Jaya Campus, 45600 Bestari Jaya, Selangor, Malaysia \\ ${ }^{3}$ Faculty of Electrical Engineering, University of Technology MARA, 23000 Dungun, Terengganu, Malaysia \\ *Corresponding author E-mail: ihsan.yassin@gmail.com
}

\begin{abstract}
This project presents a method to detect diabetic retinopathy on the fundus images by using deep learning neural network. Alexnet Convolution Neural Network $(\mathrm{CNN})$ has been used in the project to ease the process of neural learning. The data set used were retrieved from MESSIDOR database and it contains 1200 pieces of fundus images. The images were filtered based on the project needed. There were 580 pieces of images types .tif has been used after filtered and those pictures were divided into 2, which is Exudates images and Normal images. On the training and testing session, the 580 mixed of exudates and normal fundus images were divided into 2 sets which is training set and testing set. The result of the training and testing set were merged into a confusion matrix. The result for this project shows that the accuracy of the CNN for training and testing set was $99.3 \%$ and $88.3 \%$ respectively.
\end{abstract}

Keywords: Diabetic Retinopathy; Alexnet Convolution Neural Networks; MESSIDOR database; Deep learning.

\section{Introduction}

Diabetes is a serious complex condition affecting approximate 2.6 million Malaysians above 18 years old in 2012 according to National Diabetic Registry (NDR) [1]. It is a condition that requires constant monitoring and if complications develop, its can reduce life expectancy. Moreover, currently there is no cure for the diabetes. Therefore, it is very important to have some knowledge about the condition, effect on the body and how to effectively managing it. According to the tittle, the diabetes detection has been done by using fundus image and deep learning neural network. Fundus image is an image that been captured by using a colour video 3CCD camera on a Topcon TRC NW6 non-mydriatic retinography with a 45-degree field of view. Briefly, fundus is an image of interior of the human eyes.

Therefore, this project presents a method to detect a diabetic disease on a person by using the fundus images of their eyes. According to [2], diabetes can have many effects on the eye's such as fluctuation in vision clarity, flurry and/or distortions in vision, eye pain, cataracts, slower in healing after the eyes injuries and blindness. Moreover, most of the scariest things that effect to the eye of diabetes patient is the blindness. Basically, diabetic retinopathy was something that harm to the retina in the eyes. Basically, retina was laying at the back of the eyes and its sensitive to the light. Therefore, if it is damaged, its can causing someone to be a blindness.

According to [3], there were two types of diabetes which is diabetes type 1 and type 2 . However, by far, the most common form of diabetes is type 2 diabetes which is approximate $90 \%$ to $95 \%$ suffering from this disease.

\subsection{Diabetes}

Type 1 diabetes is called as insulin-dependent diabetes. It is often beginning in childhood and since it starts from childhood it used to be called juvenile-onset diabetes. Type 1 diabetes is an autoimmune condition and it's caused by the body to attacking its own pancreas with antibodies. In addition, a person that has type 1 diabetes, their pancreas will be damaged and it will not make any insulin. Basically, this type of diabetes may be caused by a genetic predisposition which is comes from their parents. It could also be the result of faulty beta cells in the pancreas that normally produce insulin. Therefore, the treatment for type 1 diabetes involves taking insulin, which needs to be injected through the skin into the fatty tissue below.

Then, for type 2 diabetes it is called as adult-onset diabetes, which is an obese and overweight kids. According to [4], teenagers nowadays were possesses with this type of diabetes. Scientifically, type 2 diabetes was called as non-insulin-dependent diabetes and it is the higher case rather than type 1 diabetes. In addition, it can make some organ didn't fully functionally, especially on the smallest blood vessels in the body which working to sustaining the nerve of kidneys and eyes. After that, it also can affect the pancreas where it is usually produces an insulin and it causes the amount of insulin produced is not enough for the body's needs, or the body's cells are resist to receive the insulin. Typically, obese persons which have height $20 \%$ lower than their ideal body are easier to have this disease because they have an insulin resistance in their body[4]. However, type 2 diabetes can be preventing by having some exercise, taking nutrition and managing their weight. Unfortunately, type 2 diabetes tends to progress and the diabetes medications are often needed. 
Basically, the working principle for the eyes to see an object, the light must be passed from front of the eye which is pupils, then iris and others. Lastly, the last will be arrived at the retina which is converting light signal to electrical signal and then the signal will be interpret by brain and produce the image. This disease mostly caused by too high blood sugar for a long period of time. Since the patients are continuously having the high blood sugar, it will be damaging the blood vessels inside of their eyes. After its damaged, the vessels will begin to leak and sometimes it will create some new vessels in the eye to recover the damaged vessels. Unfortunately, these new vessels capable of risking the vision.

\subsection{Diabetic Retinopathy}

According to $[2,3,5]$, there are two types of diabetic retinopathy which is non-proliferative diabetic retinopathy (NPDR) and proliferative diabetic retinopathy (PDR). NPDR is a sign of the early stages of diabetic retinopathy and its divided into 3 types which is Mild NPDR, Moderate NPDR and Severe NPDR. To indicate mild NPDR, it has a small area of balloon-like that swelling inside of the retina's tiny blood vessels which is scientifically called as micro-aneurysms. These micro-aneurysms are harmful and may leak some fluid into the retina. After that, typically there was a macula inside of our eyes, but for diabetic patients it was called as Diabetic Macular Edema (DME). Basically, macula is important for sharping and straight-ahead visioning and it is typically used for reading, recognizing faces, and driving. According to [4], about half of all people with diabetic retinopathy will develop these DME. Normally, DME is occurs when the diabetic retinopathy become nastier. Nevertheless, these DME can occurs at any stage of the disease.

Next, for the Moderate NPDR, it is a disease progresses where the blood vessels that nourish the retina become swell and distort. Since then, the efficiency for its to transport blood will be decreases. For Moderate NPDR and Mild NPDR, both of the conditions causing some changes to the appearance of the retina characteristic and may help the eye to have DME. Other type for NPDR is Severe NPDR, its happened when there are so much blood vessels were blocked and depriving the blood supply to areas of the retina.

After that, PDR is referring to the later stages of this disease. At this point, retina receiving limited oxygen since the blood circulation is not working properly. This is the stage that the new blood vessels begin to grow and cloud the vision. If its left untreated, it can lead to the detachment of the retina and eventual blindness. Therefore, it will cause the retina to swell, which will lead to blurred or cloudy vision. Normally, the leaking will affect both eyes.

\subsection{Fundus Image}

Fundus image is an image of an eyes which is the interior surface. This fundus images were included the retina, optic disc, macula, fovea, and posterior pole. According to [6], the fundus can be examined by using ophthalmoscopy or fundus photography. It can be captured by using fundus camera in a fundus photography or ophthalmoscopy session. To capture the fundus image, there are many equipment need to be considered. First is the fundus camera, where it is specialized low power microscope and the optical design is based on the indirect ophthalmoscope.
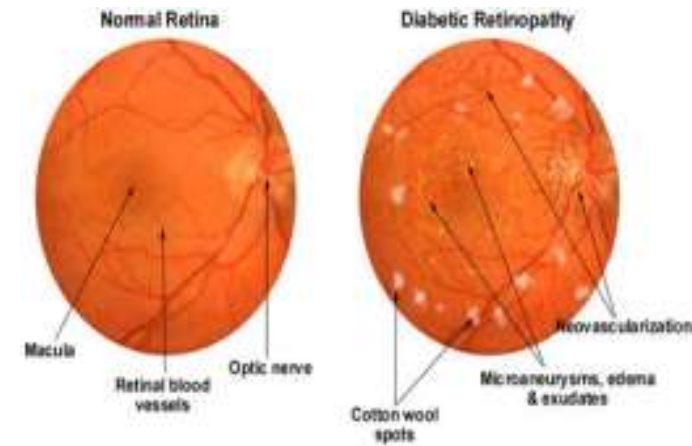

Fig. 1: Fundus Images of An Eyes.

Based on figure above, on the left side of the figure shows a normal retina and the right side is a diabetic retinopathy. At the diabetic retinopathy, it shows the neovascularization point, which is nearly to the optic nerve and the optic nerve is the brightest region of fundus images. It is appeared because of the retina blood vessels are leaked and then its growth some random abnormal blood vessels. Next on the exudates, it is divided into two types which is hard exudates and soft exudates. For the soft exudates, it can be defined as a cotton-wool spots which is a nerve fibre layer infarction from occlusion of precapillary arterioles. These cotton-wool spots are often blocked by the micro aneurysms and vascular hyperpermeability. Basically, this cotton wool appears as fluffy white patches on the retina.

For the hard exudates, it is a distinct yellow-white intra-retinal deposit which can fluctuate from small specks to larger patches and can evolve into rings known as circinate. Most of the hard exudates is contains of serum proteins, lipids, and protein which has been created by the breakdown of the blood-retina barrier. Nevertheless, the micro aneurysms are the earliest changes of diabetic retinopathy and usually looks saccular and localised capillary dilatations. Normally, they appear as small red dots in slight retinal layers and were existing to rupture the deeper layers of the retina, such as inner nuclear and outer plexiform layers. However, its relieve since this red dot did not affect the vision and it is one. After that, in [6] state that by using the fundus images, human eyes can detect the intraregional haemorrhages which is shaped as dot-blot (arrowhead), flame (arrow) and boat-shaped (asterisk) depending upon their depth within the retina. However, between the dot haemorrhages and micro aneurysms, it is hard to differentiate by using human eyes since both are changes of background retinopathy. Typically, every shape of haemorrhages is correlate to the others shape on the eyes. For dot-blot haemorrhage, it corresponds to blood in the middle layers which is can be specified as inner nuclear and outer plexiform layers of the retina. Next, the flame-shaped haemorrhage corresponds to blood in the nerve fiber layers, and the boat-shaped haemorrhage corresponds to sub hyaloid blood.

Basically, on the early stage of this disease, it is difficult to detect from the outlook view of a person. Therefore, a check-up with the doctor is needed. Typically, in Malaysia to detect this disease, a blood sugar test and urine test will be used to determine whether a person has a diabetes or not. In addition, these two methods take a lot of process and takes a long time to complete. Besides that, the methods that been applied in Malaysia is outdated. Malaysia need some improvement of their technology on medical sectors as its quite important for Malaysia to achieve well developed countries title in 2020.

Next, there are a lot of existing project that detecting a diabetes by using fundus. But, most of them were complicated where it is used some filter on the image such as colour of the image, size of the image [7]. The aim of this project is to detect an early signs of diabetes disease by using fundus images, classified by using CNN . Therefore, by using deep learning $\mathrm{CNN}$, the detection is much easier and faster. 


\section{Methodology}

\subsection{Data Collection}

The data collected was retrieved from the MESSIDOR database. The MESSIDOR database was created to evaluate different lesion segmentation methods for colour eye fundus images, in the frame work of diabetic retinopathy screening and diagnosis. It has been publicly distributed since 2008 [8]. There are 1200 eye fundus colour numerical images of posterior pole in MESSIDOR database. There were 3 ophthalmologic departments that produce these images by using a colour video 3CCD camera on a Topcon TRC NW6 non-mydriatic retinography with a 45-degree field of view. Besides that, the images have been captured using 8 bits per colour plane. The size of each images is $1440 \times 960$ pixels and ap proximately size data of the image is $4 \mathrm{MB}$ per pieces which expressed as a high resolution, larger dimension and very clear to detect the symptoms of diabetic. In Figure 2, it shows a sample of the fundus image of normal eyes and with exudates that been retrieved from MESSIDOR database. As can be seen in Figure 2(i), it shown some exudates appears as white spots on the fundus image. Nevertheless, Figure 2(ii) shown the fundus image is completely without white spots.

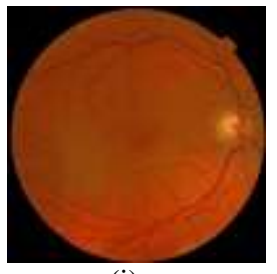

(i)

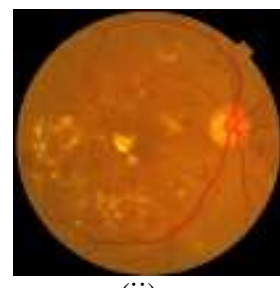

(ii)
Fig. 2: (i) Normal eyes, (ii) Exudates eyes.

\subsection{Implementation on MATLAB}

From the data collected, it has been filtered based on the image contents which is exudates and normal image. After filtering the image, there are about 580 images type tif has been collected and all these images were divided into 2 set which is normal and exudates. After that, all fundus image that contain exudates is numbered from 1 (e.g. 1, 2, 3...etc.). After that, all the normal fundus image is numbered by continuing the last number of exudates fundus images number (e.g. 340, 341, 342 ...etc.).

Before setup the training and testing process on the MATLAB, the dataset has to be arranged well to ease the process of deep learning. Therefore, firstly, the images that been numbered are merged into one folder. After that, all the images have been compressed into one file which is .mat file type. While compressing the image, the image size has been resized simultaneously to tally with the required Alexnet image input size which is $227 \times 227 \times 3$. Basically, this .mat file contains all the fundus image which is stored as a number of array and it is used in deep learning process. Therefore, without this file the deep learning process cannot be proceed.

After complete arranging the data, CNN process has to be prepared. Firstly, since this project is using Alexnet CNN template, it has to be modified in order to make the Alexnet CNN system equalize with the project systems. Basically, the Alexnet CNN template contains 25 layers which is listed on Table 1.

Table 1: Structure of CNN (modified AlexNet)

\begin{tabular}{|c|c|c|}
\hline Layers & Layers Name & Layers Description \\
\hline 1 & 'data' & Image Input \\
\hline 2 & 'conv' & Convolution \\
\hline 3 & 'relu1' & ReLU \\
\hline 4 & 'norm1' & Cross Channel Normalization \\
\hline 5 & 'pool1' & Max Pooling \\
\hline 6 & 'conv2' & Convolution \\
\hline 7 & 'relu2' & ReLU \\
\hline
\end{tabular}

\begin{tabular}{|c|c|c|}
\hline 8 & 'norm2' & Cross Channel Normalization \\
\hline 9 & 'pool2' & Max Pooling \\
\hline 10 & 'conv3' & Convolution \\
\hline 11 & 'relu3' & ReLU \\
\hline 12 & 'conv4' & Convolution \\
\hline 13 & 'relu4' & ReLU \\
\hline 14 & 'conv5' & Convolution \\
\hline 15 & 'relu5' & ReLU \\
\hline 16 & 'pool5' & Max Pooling \\
\hline 17 & 'fc6' & Fully Connected \\
\hline 18 & 'relu6' & ReLU \\
\hline 19 & 'drop6' & Dropout \\
\hline 20 & 'fc7' & Fully Connected \\
\hline 21 & 'relu7' & ReLU \\
\hline 22 & 'drop7' & Dropout \\
\hline 23 & 'fc8' & Fully Connected \\
\hline 24 & 'prob' & Softmax \\
\hline 25 & 'output' & Classification Output \\
\hline
\end{tabular}

All of these layers are not fixed and suitable on any situation of deep learning process. Therefore, some modification toward Alexnet layers has been applied which is on layer 23 and 25. On layer 23 , it is modified to define that there were only 2 class has been included in the process. On layer 25 , it is used to refresh the class been included. Below shown the modified layers.

layers $(23)$ = fullyConnectedLayer(2);

layers $(25)=$ classificationLayer;

After modifying layers, several options on training session has been applied. First option was running the training on GPU. Next, the maximum epochs (i.e. the number of training session) on the training is sets to 20 and the batch size (i.e. the number of size run in a time) is sets to 64 . After setup all the training option, the process of deep learning is processed.

On the CNN process, the dataset has been divided into 2 which is testing set and training set. On the training session, $50 \%$ (i.e. 290 pieces of fundus images) of data on the folder that containing the numbered image has been used to train the neural network. After that, another $50 \%$ data on the folder are used to testing the neural network.

\section{Results and Disscussion}

The result of this project has been simplified by using confusion matrix. The figure below shown the confusion matrix.

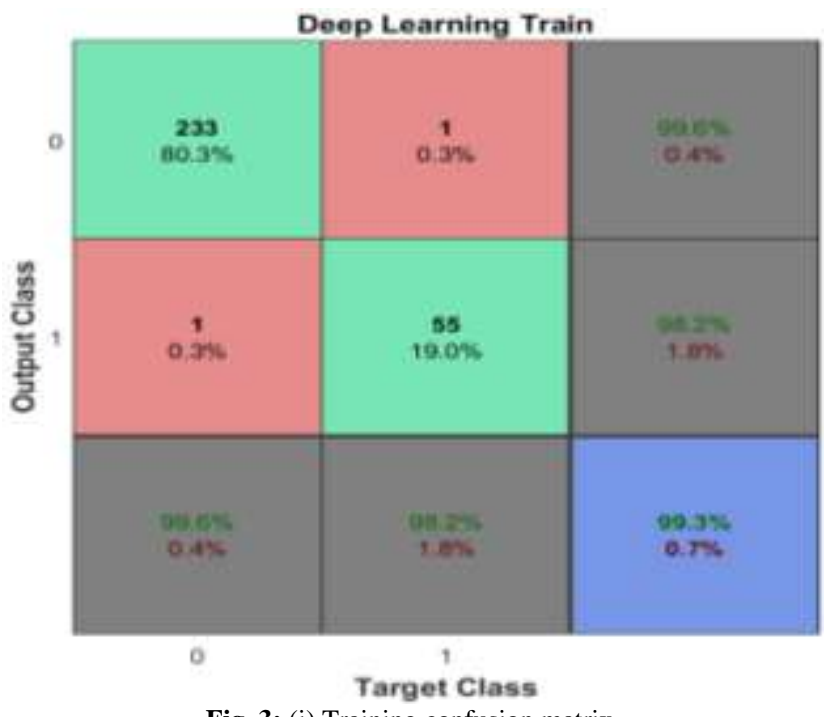

Fig. 3: (i) Training confusion matrix. 


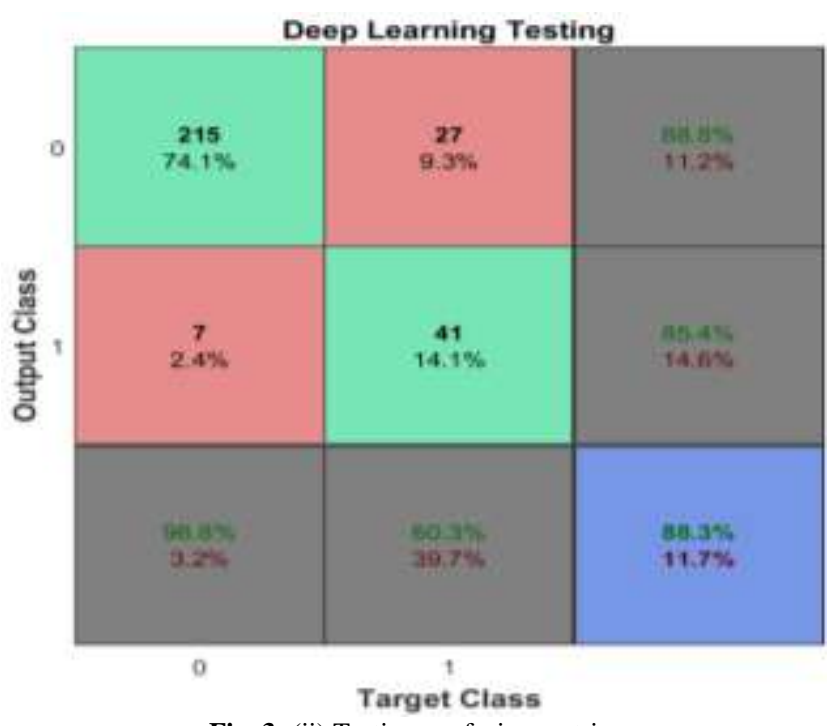

Fig. 3: (ii) Testing confusion matrix.

Based on Figure 3, it shows confusion matrix for testing data set and training data set. The testing set consist of 290 cases (i.e. 222 Normal fundus images and 68 Exudates fundus images), while on the training set consist of 290 cases (i.e. 234 Normal fundus images and 56 Exudates fundus images). Typically, the confusion matrix was divided into 2 axis which is $y$-axis indicates as output class while the $\mathrm{x}$-axis indicates as the target class. For the output class ' 0 ', it indicates that the normal fundus images eyes that been given to the $\mathrm{CNN}$. While, the output class ' 1 ' indicates the exudates fundus images that been given to CNN. For the target class ' 0 ' indicates the $\mathrm{CNN}$ targets for normal fundus images, while target class ' 1 ' indicates the $\mathrm{CNN}$ targets for exudates fundus images. Next, for the matrix [0 0] (i.e. [x-axis y-axis]), it shows the green box (i.e. True Positive) which is indicates as the correct classification that been tested by the CNN for normal fundus images. While, the matrix [ $\left[\begin{array}{ll}0 & 1\end{array}\right]$ red box (i.e. False Negative) indicates the misclassification by the $\mathrm{CNN}$ for normal fundus images. On the matrix [1 1], it shows the green box (i.e. False Positive) which is indicates as the correct classification that been tested by the $\mathrm{CNN}$ for exudates fundus images. While, the matrix [1 0 ] red box (i.e. True Negative) indicates the misclassification by the $\mathrm{CNN}$ for exudates fundus images.

Lastly, on the blue box, it indicates the accuracy of the CNN which is total of true positive and true negative divided with total of the images. Based on Figure 3(ii), the total of normal fundus image that been predicted correctly by $\mathrm{CNN}$ was 215 pieces and incorrect was 7 pieces. Next, total of exudates fundus image that been predicted correctly by CNN was 41 pieces and incorrect was 27 pieces. The accuracy of the $\mathrm{CNN}$ on the testing set was $88.3 \%$. Based on Figure 3(i), the total of normal fundus image that been predicted correctly by $\mathrm{CNN}$ was 233 pieces and incorrect was 1 pieces. Next, total of exudates fundus image that been predicted correctly by $\mathrm{CNN}$ was 55 pieces and incorrect was 1 pieces. The accuracy of the CNN on the testing set was $99.3 \%$.

Basically, to determine whether the deep learning was success or not is based on the accuracy of the CNN. Therefore, based on the testing set, it shows that the accuracy was $88.3 \%$ while on the training sets the accuracy was $99.3 \%$ which is approximately $100 \%$. Besides that, on the testing confusion matrix it, CNN shows 27 images has been misclassified as the normal fundus images while the images were exudates images. After that, $7 \mathrm{im}$ ages have been classified as an exudates fundus images while the images were normal fundus images. Therefore, this CNN are bias towards classifying an exudates fundus images as a normal fundus images rather than classifying a normal fundus images as an exudates fundus images.

There are several factors that the causing accuracy $88.3 \%$. One of the factors is when resizing the image. While resizing the image, some the image has been cut-out and maybe the image that has been cut-out carrying an important info to the CNN. After that, another reason that the accuracy is not approximately $100 \%$ is because the CNN was insufficient of data to train the neural. Lastly, it may cause by the smaller exudates appears on the images. When the exudates were too small, it is difficult for the CNN to detect the exudates on the images.

Briefly, to conclude this project, even the $\mathrm{CNN}$ accuracy for testing is $88.3 \%$ it can be considered as a success CNN since the accuracy is higher than $85 \%$. After that, $\mathrm{CNN}$ accuracy for training can be considered as a success CNN since it is approximately $100 \%$ accuracy.

\section{Conclusion}

This project successfully detects the diabetes by using deep learning on a fundus images and it can be used as one of method to detect the diabetes on the future. Nevertheless, it need some improvement to make the accuracy of the project nearest to $100 \%$. Next, Alexnet layers is the most perfect layer for deep learning neural network these day.

\section{Acknowledgement}

Authors gratefully acknowledge the financial support from Ministries of Higher Education Malaysia and Institute of Research Management and Innovation (IRMI) Universiti Teknologi MARA Grant No: 600-RMI/NRGS 5/3 (3/2013).

\section{References}

[1] Feisul, M. I., \& Azmi, S. (2013). National diabetes registry report. 2009-2012. Ministry of Health Malaysia.

[2] Neuwirth, J. (1988). Diabetic retinopathy: What you should know. https://nei.nih.gov/sites/default/files/Diabetic-Retinopathy-WhatYou-Should-Know-508.pdf.

[3] Vislisel, J., \& Oetting, T. (2010). Diabetic retinopathy: From one medical student to another. http://eyerounds.org/tutorials/diabeticretinopathy-med-students/Diabetic-Retinopathy-medicalstudents.pdf.

[4] Diabetes UK. (2006). What is diabetes. http://www.godnaturalcures.com/healthc/PDF\%207\%20EBOOKS/ natural-help-for-diabetes.pdf.

[5] Antonetti, D., Klein, R., \& Gardener, T., Diabetic retinopathy. (2012). https://www.aoa.org/patients-and-public/eye-and-visionproblems/glossary-of-eye-and-vision-conditions/diabeticretinopathy.

[6] Ophthalmic Photographers' Society. Fundus photography overview. https://www.opsweb.org/page/fundusphotography.

[7] Poornima, S. V., Nishchala, T. K., \& Umamakeswari, A. (2014). Detection of diabetic retinopathy by applying total variation. Biomedical Research, 25(4), 560-563.

[8] Decencière, E., Zhang, X., Cazuguel, G., Lay, B., Cochener, B., Trone, C., P. Gain, R. Ordonez, P. Massin, A. Erginay, \& Charton, B. (2014). Feedback on a publicly distributed image database: The Messidor database. Image Analysis and Stereology, 33(3), 231-234. 